\section{Avaliação das unidades de coleta do Programa de Triagem Neonatal no Estado do Rio de Janeiro}

\section{Evaluation of Neonatal Screening Program collection centers in the State of Rio de Janeiro}

Judy Botler 1

Luiz Antonio Bastos Camacho 2

Marly Marques da Cruz 3

\section{Abstract}

Objectives:evaluate the structure and process of collection centers with focus on specimen collection, transportation of tests and active search in the state of Rio de Janeiro's Neonatal Screening Program (PTN-RJ).

Methods: 66 of the 422 collection centers were chosen, according to type, location and workload volume. The evaluation tool employed during the research included: semi-structured questionnaires, guidelines for direct observation and document review. Scores were generated for both structure and process components assessment at each collection center. Median scores were then calculated, evaluated for internal analytical consistency, score correlation between structure and process components.

Results: the overall median score (maximum of 100) was 69.1 (variation: 50.5-86.6) for structure and 63.9 (variation 46.9-77.4) for process components. The performance of each collection center showed consistency among centers with similar structure. Maternity centers and Family Health Program Units (PSF) showed superior performance in their process components, with collection centers type B (UCB) showing lower performance with the same group of components. The Cronbach alpha coefficient indicated a high consistency of among the assessment tools for structure (alpha $=0.93)$ and process components (alpha=0.81). The structure and process components scores showed weak correlation (Pearson coefficient $=0.44$; Spearman $=0.42$ ). Differences in mean scores among types of collection center showed considerable magnitude, but without statistical significance $(p=0.387)$.

Conclusions: the overall performance in structure and process components was satisfactory. Deficiencies were found in professional training and in availability of specimen collection and educational material.

Key words Neonatal screening, Evaluation, Program evaluation
1-3 Escola Nacional de Saúde Pública Sérgio Arouca. Fundação Oswaldo Cruz. Rua Leopoldo Bulhões, 1480. s1.820. Manguinhos. Rio de Janeiro, RJ, Brasil. CEP: 21.041-210.

E-mail: jbotler@ensp.fiocruz.br

\section{Resumo}

Objetivos: avaliar estrutura e processo das unidades de coleta (UC) e incluindo coleta e transporte das amostras, e busca ativa do Programa de Triagem Neonatal do Estado do Rio de Janeiro (PTN$R J)$.

Métodos: foram selecionadas 66 das 422 UC existentes, conforme tipo, localização e volume de amostras. Foram aplicados questionário semi-estruturado e roteiros para observação direta e análise documental. Foram gerados escores para estrutura e processo para cada UC. Foram calculadas medianas da pontuação, avaliada a consistência interna do instrumento, correlação de escores entre estrutura e processo.

Resultados: o escore mediano global (máximo de 100) foi de 69,1 (variação: 50,5-86,6) para estrutura e 63,9 (variação: 46,9-77,4) para processo. O desempenho das UC foi semelhante em estrutura. Maternidades e PSF tiveram desempenhos superiores em processo, e UCB inferiores. O coeficiente alfa de Cronbach indicou alta consistência dos instrumentos para avaliação de estrutura (alfa $=0,93)$ e processo (alfa $=0,81)$. Os escores de estrutura e processo apresentaram fraca correlação (coeficiente de Pearson = 0,44; Spearman $=0,42$ ). Diferenças nos escores médios entre tipos de UC demonstraram considerável magnitude, porém sem significância estatística $(p=0,387)$.

Conclusões: o desempenho global em estrutura e processo foi satisfatório. As deficiências foram encontradas na capacitação de profissionais e suprimento de material para coleta e educativo.

Palavras-chave Triagem neonatal, Avaliação, Avaliação de programas 


\section{Introdução}

Os programas de triagem neonatal (PTN) detectam diversas doenças assintomáticas em recém-natos a tempo de prevenir sequelas ao seu desenvolvimento. Amostras de sangue são coletadas em unidades de coleta (UC) e analisadas em laboratório central. Resultados alterados requerem confirmação diagnóstica e tratamento. Este fluxo envolve diversas etapas, profissionais e instituições, com capacitação e envolvimento diferenciados. O alcance das metas exige articulação ágil e oportuna do sistema.

O Brasil introduziu a triagem neonatal como um programa de saúde pública em $2001^{1}$ para quatro doenças: fenilcetonúria (PKU), hipotireoidismo congênito (HC), hemoglobinopatias e fibrose cística. O PTN brasileiro prevê acesso universal da triagem ao tratamento. Os 27 Estados foram credenciados em fases conforme sua capacidade de atender a todas as etapas necessárias às doenças previstas. A Fase I inclui PKU e HC; a Fase II com PKU, HC e hemoglobinopatias; e a Fase III contempla as quatro condições.

Em cada Estado, o Serviço de Referência em Triagem Neonatal (SRTN) articula as atividades entre UC, laboratório e ambulatório. ${ }^{2} \mathrm{Na}$ concepção do PTN governamental previu-se uma etapa de avaliação ainda não executada. Em outros países, a avaliação dos PTN vem ganhando importância, ${ }^{3}$ com iniciativas como o Performance Evaluation and Assessment Scheme (PEAS), ${ }^{4}$ desenvolvido para auto-avaliação dos PTN norte-americanos, 2,3 e cobrindo todas as etapas do sistema.

O PTN do Estado do Rio de Janeiro (PTN-RJ), criado na década de 1980, conta com 422 UC distribuídas por 92 municípios. Foi credenciado para a Fase II do programa governamental com três SRTN: um instituto de endocrinologia (denominado "SRTN A"), uma entidade filantrópica ("SRTN B") e um hospital municipal do RJ ("SRTN C").5,6 Em 2005, o SRTN C desativado transferiu sua demanda para o SRTN A. Os SRTN A e B dividiam a demanda do Estado com fluxos independentes. O instituto de hematologia (IH) apoiava ambos no diagnóstico e tratamento dos casos com hemoglobinopatias.

Em 2007, o PTN-RJ alcançou cobertura de $80,4 \%$, com $42 \%$ dos testes colhidos até sete dias de vida. A mediana de tempo para confirmação diagnóstica foi de 48 dias, e $25 \%$ dos casos ultrapassa 60 dias. ${ }^{7}$ A avaliação das UC remete à discussão sobre acesso aos serviços de saúde como dimensão de desempenho desses sistemas. ${ }^{8}$ Os resultados abaixo das metas suscitaram a necessidade da avaliação.

Este trabalho, realizado em UC do PTN-RJ, teve como objetivo avaliar elementos de estrutura e processo, com ênfase nas etapas de coleta, transporte dos espécimes aos laboratórios, busca ativa para segunda coleta e confirmação diagnóstica dos casos com resultados alterados. A análise dos fatores que afetaram o acesso ao teste e a cobertura buscou subsidiar os gestores com informações úteis ao melhor desempenho dessa etapa do programa.

\section{Métodos}

Este trabalho compõe uma pesquisa avaliativa baseada em estudo de caso, cujo objeto é o PTN-RJ com dois níveis de análise - UC e SRTN. O primeiro nível envolve grande número de unidades de análise permitindo abordagem quantitativa. O segundo, com duas unidades de análise, teve enfoque qualitativo discutido em outra publicação.

A amostra de conveniência de UC de uma lista fornecida pelos SRTN foi selecionada segundo três critérios: geográfico, tipo de UC e volume de testes em 2007. Pelo critério geográfico, utilizaram-se oito regiões administrativas (RA) do Estado e cinco áreas programáticas (AP) em saúde do município do RJ, totalizando treze áreas. No critério quantitativo priorizaram-se as UC com maior volume de espécimes em 2007. A amostra totalizou 66 UC. Seis tipos de UC foram definidos conforme sua natureza administrativa: Hospital Geral (HG - 8/66), Maternidades (MT - 7/66), Programa Saúde da Família (PSF - 8/66), Postos de Saúde Estaduais (PSE - 20/66), Postos de Saúde do Município do RJ (PSM - 10/66), e um tipo denominado UCB vinculado ao SRTN B (8/66), com número menor de coletas.

$\mathrm{Na}$ coleta de dados, quatro UC foram substituídas - duas por recusa, uma por greve e uma por interrupção das atividades antes da pesquisa. Onde não havia maternidades e PSF, houve 17 substituições por PSE da mesma região. A maior participação deste tipo de UC na amostra é coerente com sua representatividade no PTN-RJ.

Os quatro níveis da avaliação foram abordados em módulos distintos no instrumento de coleta de dados (Figura 1). As técnicas para coleta de dados incluíram questionário semi-estruturado, roteiro de observação, análise documental e registro de ocorrências. Os questionários foram aplicados a informantes-chaves conforme a sua experiência de acordo com o nível de avaliação correspondente. No roteiro de observação, eram coletados dados da estrutura e processo que eram observados pelo coletador de dados, mas que não eram informadas pelo informante-chave. Na análise documental, foram exami- 
Representação esquemática do instrumento de coleta de dados, conforme os níveis de avaliação (módulos), técnicas de coleta de dados e informantes-chave.

\begin{tabular}{|c|c|c|}
\hline Informantes-chave & Módulos - Níveis da avaliação & Técnicas de coleta de dados \\
\hline $\begin{array}{c}\text { Coordenador / } \\
\text { Analista de sistemas, etc }\end{array}$ & Administração (A e B) & \\
\hline $\begin{array}{c}\text { Profissional coleta / } \\
\text { busca ativa }\end{array}$ & $\begin{array}{l}\text { Coleta, busca ativa e trans- } \\
\text { porte das amostras } \\
\text { (unidades de coleta) }\end{array}$ & $\begin{array}{l}\text { - Questionário semiestruturado } \\
\text { - Observação direta }\end{array}$ \\
\hline \begin{tabular}{|c|} 
Chefe do laboratório / \\
Responsável pela capacitação / \\
técnicos, etc
\end{tabular} & $\begin{array}{l}\text { SRTN } \\
(A \text { e } B)\end{array}$ & $\begin{array}{l}\text { - Análise de registros de } \\
\text { ocorrência }\end{array}$ \\
\hline & SH & \\
\hline Assistente social & Busca ativa ( $A$ e $B$ ) & \\
\hline
\end{tabular}

nados os dados de mapas de trabalhos, documentos da rotina e registros das bases de dados. O registro de ocorrências serviu para anotações sobre acontecimentos imprevistos que pudessem ter interferido na rotina, e sobre as providências tomadas.

$\mathrm{Na}$ construção do instrumento de coleta de dados foram incluídas perguntas do PEAS, 4 roteiro de cadastramento de SRTN, 9 manual de normas técnicas e operacionais do PTN governamental2 e diretrizes internacionais.10-18 Os quatro módulos foram denominados: 1) Administração; 2) Coleta, busca ativa e transporte das amostras"; 3) "SRTN" e 4) "Busca ativa", que incluem as diversas etapas do PTN, que vão da coleta do teste até a confirmação diagnóstica. Nesta avaliação, o segundo módulo, foi aplicado aos profissionais responsáveis pelas coletas e busca ativa. Os demais módulos foram aplicados na avaliação dos SRTN e serão discutidos em outra publicação.

Este módulo foi testado num estudo-piloto em UC não selecionada na amostra de conveniência. A consistência interna do questionário foi analisada e medida com o coeficiente alfa de Cronbach.

Os questionários foram aplicados por duas profissionais de saúde a informantes-chaves após assinatura de Termo de Consentimento Livre e Esclarecido.

Os dados foram tabulados em planilha eletrônica MS-Excel ${ }^{\circledR}$ com as UC agrupadas por tipos. Os elementos com maior número de itens a serem avali- ados tiveram peso maior na pontuação. Perguntas de estrutura, processo e questões descritivas foram analisadas em planilhas distintas.

A soma das respostas que indicavam cenários desejáveis gerou uma pontuação máxima possível (100). Foi criado um escore segundo a fórmula: [total de pontos observados/ pontuação máxima possível] x 100. Em todas as UC, considerou-se a pontuação máxima possível. Em apenas três casos desconsiderou-se observação da coleta e/ou da geladeira e recalcularam-se os escores com novo denominador.

Em 11 UC (um UCB, três maternidades, três hospitais gerais, duas UC do PSF e duas UC do PSE) não foi possível observar coletas. Em uma unidade de coleta tipo B (UCB) e em dois PSE não houve acesso à geladeira onde havia testes armazenados. Nestes casos, os escores de estrutura e processo foram recalculados atendendo a estas diferenças sem penalizá-los na comparação.

Os dados foram analisados com o aplicativo estatístico SPSS 19 para cálculos de mediana dos escores de estrutura e processo, global e por tipo de UC, identificação em cada tipo de UC dos extremos superior e inferior de desempenho, e cálculo da correlação entre os escores de estrutura e processo (coeficientes de correlação linear de Pearson e de Spearman, antecipando desvio da distribuição normal). A significância estatística das diferenças nas distribuições dos escores foi avaliada pelo teste 
de Kruskal-Wallis.

As respostas iguais eram agrupadas em cada tipo de UC e, posteriormente, era calculada a proporção de cada tipo de resposta para cada tipo de UC. Após o cálculo de correlação entre estrutura e processo, foram selecionadas as quatro UC com melhor e as quatro com pior desempenho. Estas foram analisadas como tipos de UC à parte para efeito de comparação com os demais tipos de UC.

Para definir o nível de desempenho das UC foram utilizados como parâmetros os tipos de UC com desempenho mais fraco e aquelas com melhor desempenho. Ou seja, as mais fracas foram aquelas que se aproximavam mais do tipo "FD" (fraco desempenho), enquanto as de melhor desempenho foram as que estavam mais próximas do tipo "BD" (bom desempenho), conforme Tabela 1. As comparações não dependeram de um ponto de corte porque foram baseadas nos valores extremos (melhor e pior desempenho).

A pesquisa foi aprovada nos Comitês de Ética em Pesquisa (CEP) da Escola Nacional de Saúde Pública - Fiocruz (Protocolo CEP-ENSP No 113/08), Instituto Estadual de Endocrinologia (Parecer CEPIEDE No 48/08), Secretaria Municipal de Saúde do RJ (Protocolo N ${ }^{\circ}$ 265/08), um Instituto de Pediatria e Puericultura - (Cadastro FR268284), um Hospital Geral (Protocolo No 005/2009) e pelo conselho superior da instituição filantrópica. O CEP do Instituto de Hematologia aprovou, embora optasse por não participar da pesquisa. A utilização do PEAS foi autorizada pelo National Newborn Screening and Genetics Resource Center.

\section{Resultados}

O coeficiente alfa de Cronbach indicou alta consistência interna dos componentes de estrutura $(\mathrm{alfa}=0,93)$ e processo $(\mathrm{alfa}=0,81)$. Os escores de estrutura e processo mostraram fraca correlação linear (Figura 2), com coeficiente de Pearson igual a 0,44 e de Spearman igual 0,42.

A mediana global de desempenho em estrutura das 66 UC foi de 69,1 pontos (variação: 50,5 - 86,6). A mediana de processo foi de 63,9 pontos (variação: 46,9 - 77,4). Quando discriminadas por tipo, as medianas de desempenho de estrutura variaram entre 61,9 (HG) e 74,2 pontos (UCB) e as de processo entre 58,1 (HG) e 72,6 pontos (PSF). Embora de magnitude considerável, as diferenças na pontuação não alcançaram significância estatística $(p=0,387)$ provavelmente em razão do limitado tamanho da amostra.

A Figura 3 mostra distribuição homogênea dos escores de estrutura entre os tipos de UC. A figura sugere ainda que Maternidades e PSF tiveram melhor desempenho em processo e UCB apresentaram mais problemas.

As UC com melhor desempenho estavam entre as quatro com desempenho superior na correlação entre estrutura e processo (Figura 2) e maior pontuação em estrutura e processo para cada tipo (Figura 3). As quatro UC com "fraco desempenho" estavam no extremo inferior da correlação (Figura 2) e pontuação mínima nos respectivos tipos para estrutura e processo (Figura 3 ).

Nas Tabelas 1 e 2 estão apresentados os itens selecionados pela relevância para estrutura e processo em triagem neonatal, e as proporções de respostas mais adequadas em cada item de estrutura e processo, segundo os tipos de UC, e naquelas dos extremos (bom e fraco desempenho) de desempenho global. As colunas "BD" e "FD" nas tabelas possibilitam visualizar o desempenho das melhores e piores UC em cada um dos itens de estrutura e processo.

Nas UC vinculadas ao SRTN A, predominavam técnicos de enfermagem ( $46 \%$ dos $\mathrm{HG}, 57 \%$ das maternidades, $88 \%$ dos PSF, $95 \%$ dos PSE, e $80 \%$ dos PSM), enquanto nas UCB não havia categoria dominante $(29 \%$ de médicos, $29 \%$ de enfermeiros, $29 \%$ de agentes de saúde, $29 \%$ de farmacêuticos).

A maioria dos profissionais orientava sobre coletas, doenças detectadas, prazos para coletas, $2^{\mathrm{a}}$ coletas, confirmação diagnóstica e tratamento. Em apenas $2 / 7$ das maternidades e 1/10 dos PSM, havia orientação sobre coleta, em $3 / 7$ das maternidades sobre prazos, e 16/20 dos PSE sobre $2^{\mathrm{a}}$ coleta quando havia transfusões (Tabela 1).

$\mathrm{Na}$ maioria das UC, os mesmos profissionais faziam coleta e busca ativa $(\mathrm{UCB}=62,5 \% ; \mathrm{HG}=$ $61,5 \%$; maternidades $=57,1 \%$; PSE $=65,0 \%$; e PSM $=65,0 \%)$, porém no PSF esta proporção era menor $(37,5 \%)$.

Em geral, faltava material educativo como: manuais sobre coleta e busca ativa; cartazes informando o momento ideal para coleta; material educativo para pais; avisos sobre horários de coletas ou entrega de resultados. Nunca houve suspensão de coletas por falta de material em todas as UCB e $11 / 13 \mathrm{HG}$, enquanto isso ocorreu em metade dos PSM e maternidades (Tabela 1).

Em geral, nomes de mães, datas de nascimento, endereços e telefones eram cadastrados. Nomes de crianças não eram registrados em $29 \%$ das maternidades, $35 \%$ dos PSE e $43 \%$ dos HG (Tabela 1). Prematuridade, intercorrências nos período perinatal e transfusões não eram cadastradas nos HG, PSE e 


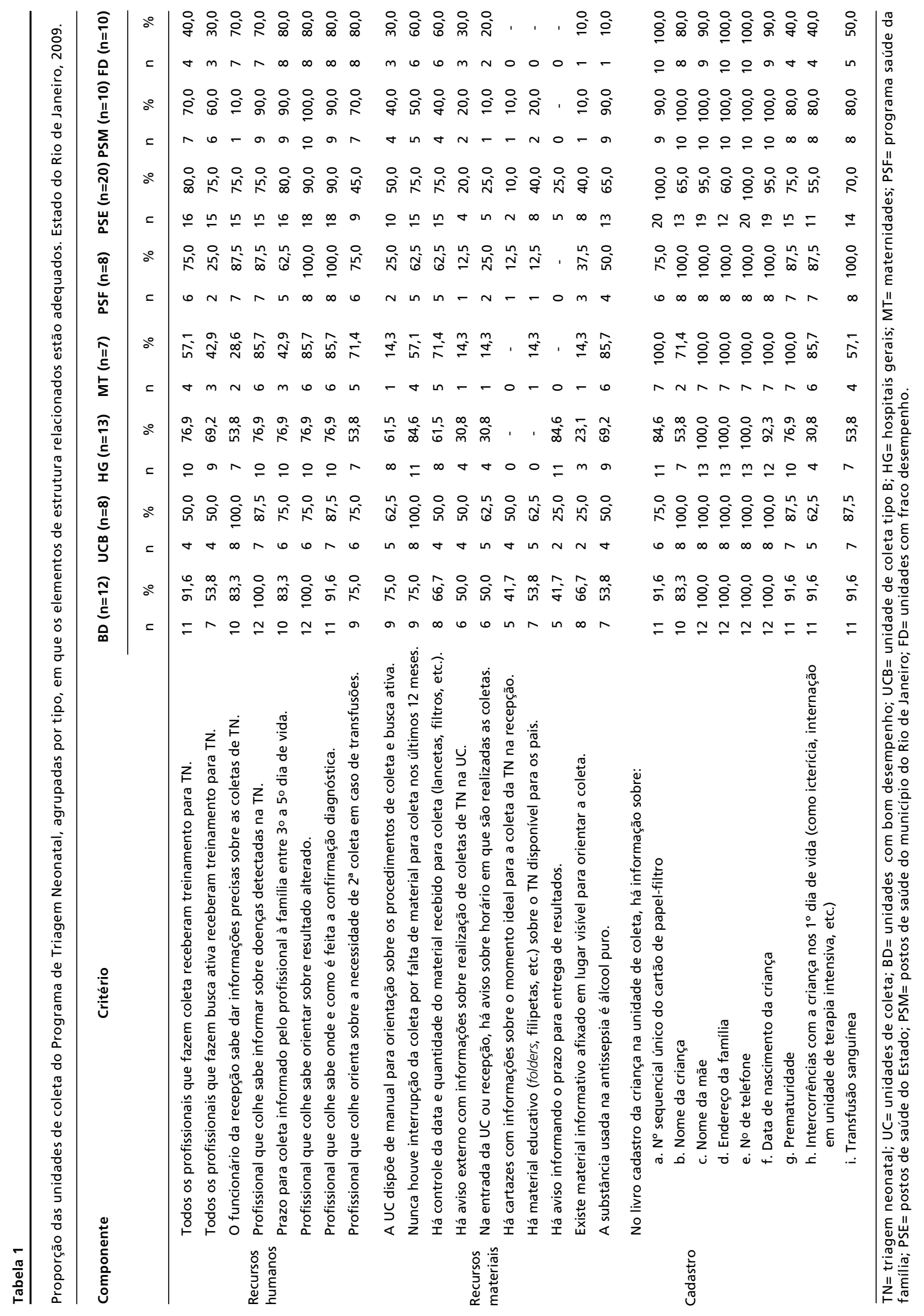


Correlação entre escores de estrutura e processo das unidades de coleta de testes de triagem neonatal no Estado do Rio de Janeiro em 2009.

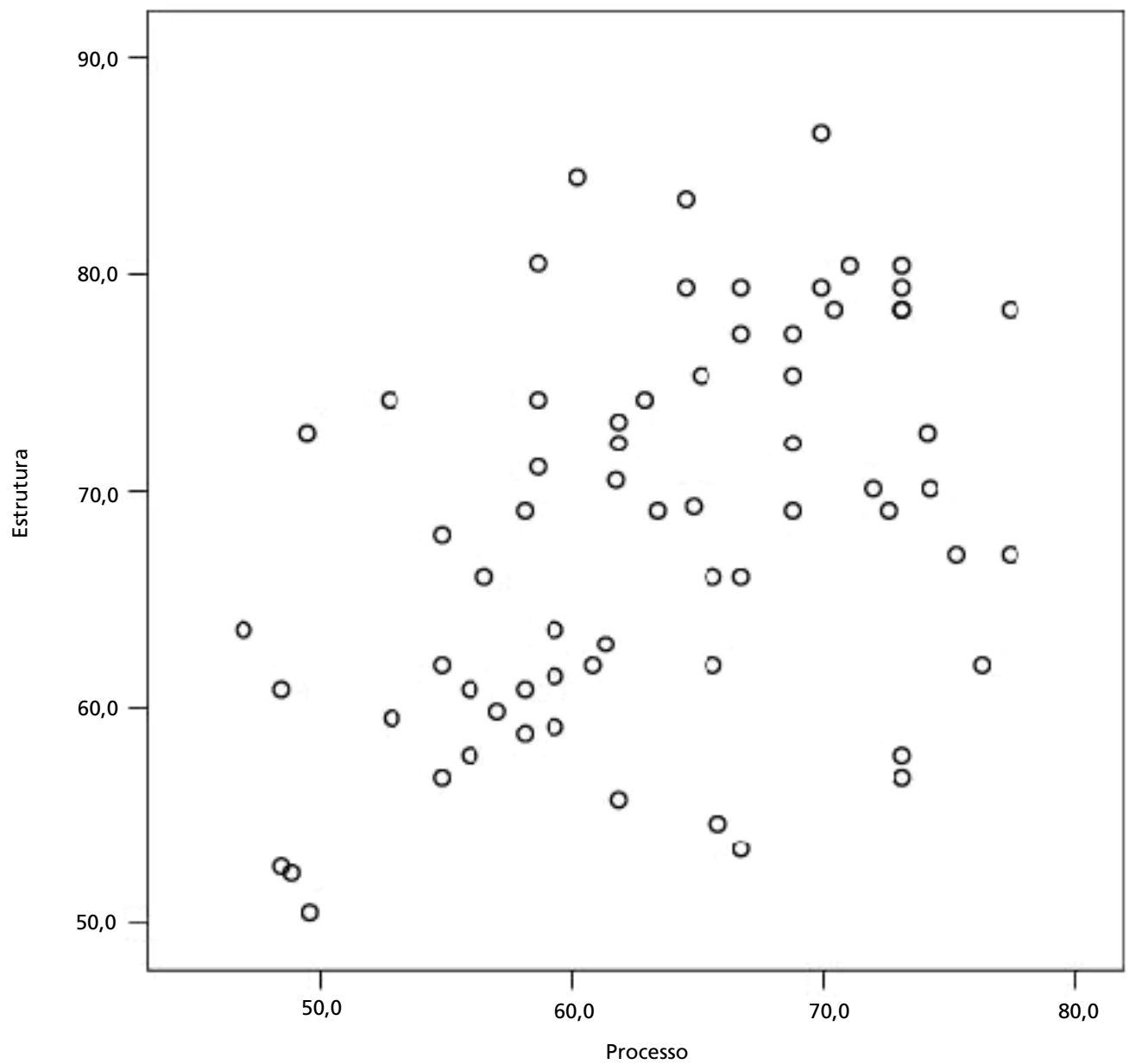

Coeficiente de Pearson $=0,44 ;$ Coeficiente de Spearman $=0,42$.

UC com fraco desempenho.

$\mathrm{Na}$ maioria das UC, as coletas ocorriam menos de cinco vezes na semana (exceto nos PSM do RJ), onde havia coletas por menos de quatro horas diárias, sem tolerância fora do horário regulamentar. Crianças residentes em outras áreas eram bem recebidas pela UC vigente (Tabela 2).

A informação sobre triagem neonatal era habitualmente transmitida no período neonatal: $69,2 \%$ dos HG; $71,4 \%$ das maternidades; $80 \%$ dos PSE e $70 \%$ dos PSM (dados não incluídos na Tabela 2). O mesmo ocorria no pré-natal em $75 \%$ das UCB e $100 \%$ dos PSF (dados não incluídos na Tabela 2). Com exceção da metade das UCB, a maioria dos pais era orientada sobre prazos adequados para as coletas.

Em metade das UCB e PSM havia segunda coleta em casos de transfusão. A verificação da coleta em crianças internadas ocorria somente na maioria dos HG, maternidades e metade dos PSF (Tabela 2).

Na maioria das UC, havia álcool puro para antissepsia, mas em metade das UCB e PSF utilizavamse outros antissépticos (Tabela 1). A secagem após antissepsia era feita com muito maior frequência no PSF, PSE e PSM do que nos demais tipos de UC (Tabela 2).

As laterais do calcanhar eram puncionadas na maioria das UC, exceto em 7/13 HG e 4/7 mater- 
Escores de estrutura e de processo das unidades de coleta de triagem neonatal do Estado do Rio de Janeiro, agrupadas por tipo de unidade, em 2009.
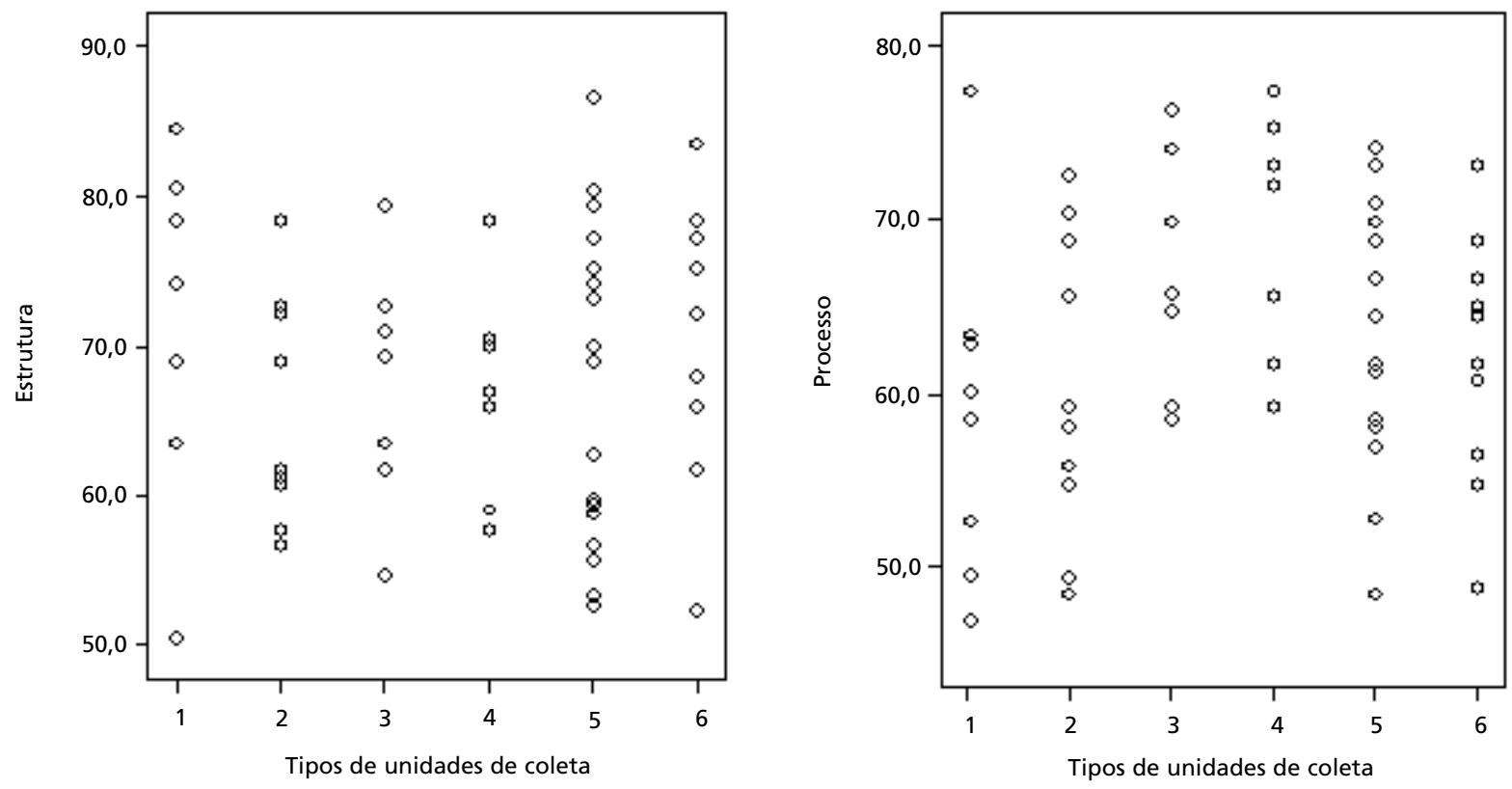

1 = unidade de coleta do tipo $\mathrm{B} ; 2$ = hospitais gerais; 3 = maternidades; 4 = programa saúde da família; 5 = postos de saúde do Estado; $6=$ postos de saúde do município do Rio de Janeiro.

nidades, onde o procedimento era inadequado. Em geral, monitorava-se a qualidade do sangue colhido, exceto em metade das UCB e maternidades. Somente na metade das UCB não havia secagem adequada das amostras, nem por tempo suficiente (37,5\% por menos de duas horas) (Tabela 2$)$.

As amostras ficavam armazenadas entre um e três dias em todos os tipos de UC, exceto em $40 \%$ das PSE que mantinham por mais tempo e em temperatura ambiente. Nesta questão houve incongruência entre informante-chave e observação - em várias UC, os profissionais ignoravam a existência de espécimes encontrados na geladeira. (Tabela 2).

O transporte de amostras ao laboratório via postal ocorria em $76,9 \%$ dos HG, $100 \%$ das maternidades, $50 \%$ das UC do PSF e $95 \%$ dos PSE. Nas UCB (75\%) e PSM (60\%) o envio era via portador. A maioria das UC anexava listagens das amostras e registrava data de envio. Em 10/13 HG, 5/8 UC do PSF e em 11/12 das UC que tiveram bom desempenho o envio ocorria duas vezes por semana. Nas demais, esse intervalo era maior. Houve poucas interrupções no transporte nos 12 meses anteriores a pesquisa (Tabela 2).

O fluxo do SRTN A previa transporte das amostras duas vezes na semana, tido como aceitável pelo manual de normas técnicas e operacionais do PNTN, 2 embora considerado longo pelas demais diretrizes. ${ }^{15,16,20}$ A maioria das UC enviava em prazo superior, incluindo as $\mathrm{UCB}$, coerentes com o fluxo estabelecido pelo SRTN B, que previa transporte semanal.

A proporção de resultados disponíveis até 15 dias após coleta era muito baixa em geral. A maioria de UC controlava as datas de reconvocação e comparecimento para segunda coleta, exceto na metade das maternidades e HG. Somente as UCB, PSE e PSF verificavam o comparecimento para confirmação diagnóstica. 


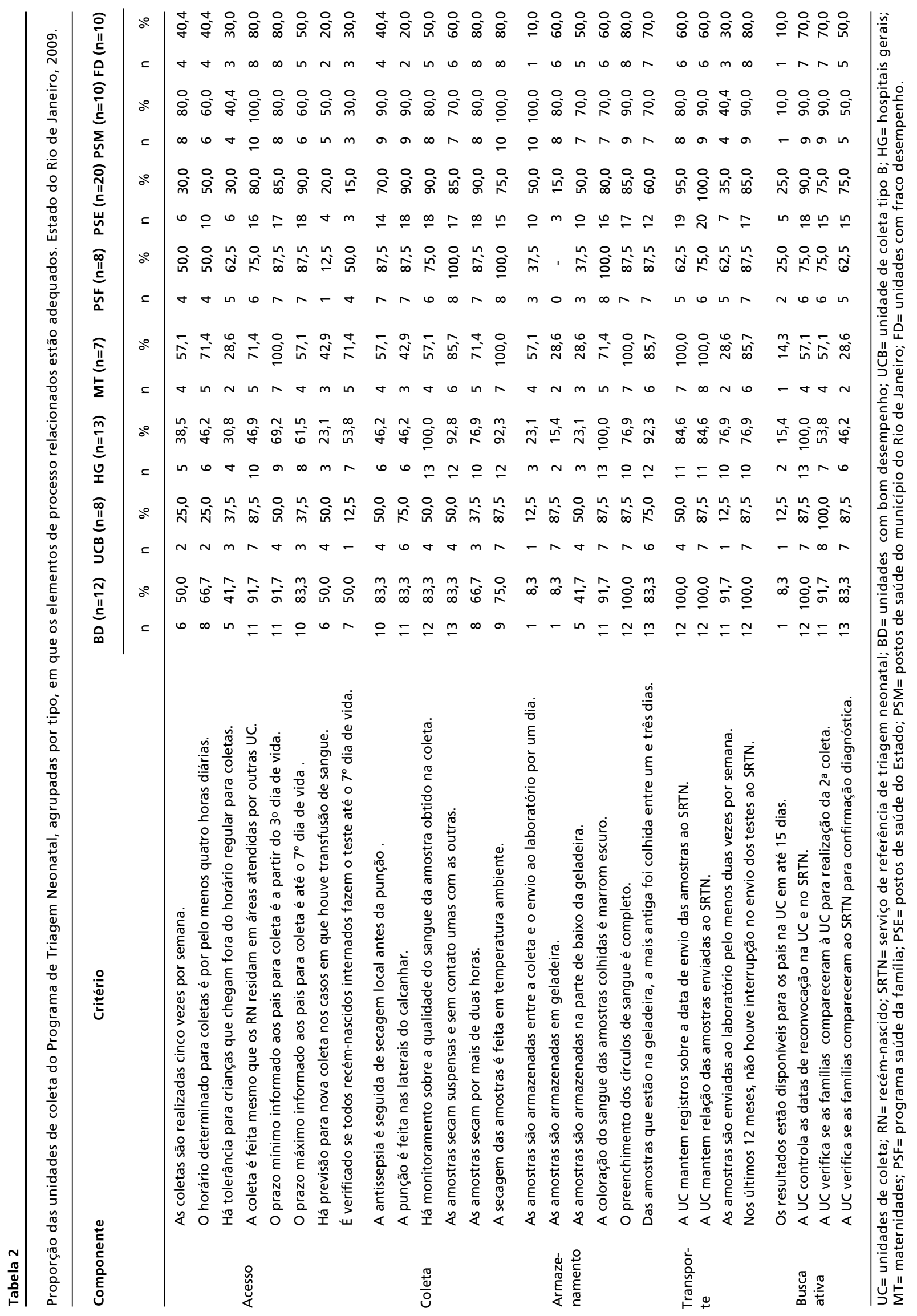




\section{Discussão}

Com características peculiares, o programa brasileiro demandou a elaboração de um instrumento de avaliação adequado às suas particularidades. $\mathrm{O}$ PTN-RJ está consolidado e integrado às ações básicas de saúde, com coberturas crescentes apesar da ligeira queda em 2007.7 Com ações gravitando entre dois SRTN, a avaliação da estrutura e processo, considerando dois sistemas organizacionais, se impôs e forneceu elementos para compreender discrepâncias apontadas em estudos recentes nos intervalos de tempo e na frequência de detecção.7,21

Os resultados indicam que muitos dos problemas encontrados se relacionam ao processo de trabalho dos profissionais, fundamentais nesse intrincado cenário. As deficiências são coerentes com o contexto do sistema de saúde em que o programa foi construído. Os problemas de informação aos usuários, coletas inadequadas, armazenamento impróprio das amostras e envolvimento insuficiente na busca ativa sugerem a necessidade de capacitações e supervisões melhores e mais frequentes. $\mathrm{O}$ foco nos profissionais deve ser compatível com o seu papel de multiplicadores da informação e a responsabilidade pela qualidade da amostra colhida no momento ideal, além de ações estratégicas no prénatal induzindo coletas mais precoces.

A coleta de amostras para teste envolve procedimentos simples, porém críticos para as etapas subsequentes e com tempo curto para realização. A recomendação para coleta entre o terceiro e quinto dia de vida da criança2,10,15,16 se apoia em evidências científicas de que o tratamento iniciado até duas semanas evita danos ao desenvolvimento.11,16,22 Em todos os níveis do PTN-RJ, identificou-se carência de material informativo e capacitação deficiente. Informações impróprias resultam em perdas de oportunidades de coleta. Coletas tardias ou amostras de má qualidade implicam em necessidade de recoletas, conduzindo a diagnósticos tardios. Resta, assim, um intervalo muito curto entre coleta e tratamento.

As maternidades seriam locais de eleição para se evitar perdas de oportunidades e garantir coletas oportunas. Contudo, especula-se que no Estado do RJ, haja carência de leitos gerando grande rotatividade, altas hospitalares precoces e coletas tardias em unidades externas. Gestantes oriundas de diversos municípios buscariam maternidades do município do RJ para, após o parto, retornarem às suas cidades, dificultando a busca ativa. Nestes casos, seria recomendável aos profissionais que atendem as famílias na alta estarem aptos a orientar sobre o momento e local para realização do teste.
Nesta avaliação, as maternidades apresentaram problemas de capacitação semelhantes aos das demais UC. As famílias só tomaram conhecimento sobre triagem neonatal na maternidade após o nascimento, possivelmente favorecendo coletas tardias que poderiam ser evitadas pela informação no prénatal.

Apesar do consenso com relação aos itens do cadastro, algumas UC não consideravam situações como prematuridade, intercorrências no período neonatal e transfusão sanguínea. A Academia Brasileira de Medicina 12 e o Manual de Normas e Técnicas e Rotinas Operacionais ${ }^{2}$ recomendam a segunda coleta para crianças prematuras em sete dias. As evidências científicas demonstram que estes casos podem simular diagnósticos falso-negativos. 12 Transfusões no período neonatal podem mascarar diagnósticos de hemoglobinopatias. Nestes casos, está prevista segunda coleta em 90 dias para preservar a sensibilidade do programa e evitar perdas de casos. 2

A carência de material educativo e a capacitação inadequada comprometeram o trabalho dos profissionais, refletindo na qualidade da informação ministrada, procedimentos de coleta e armazenamento apropriado dos espécimes.2,14,16,20 Amostras mantidas sem refrigeração em locais com temperatura ambiente elevada podem comprometer a estabilidade dos analitos.2,20

A estrutura organizacional das UC deve acolher as famílias sem perder oportunidades de coleta, agilizando o processo subsequente da triagem neonatal. Nesta avaliação, analisou-se o acesso aos testes sob diferentes perspectivas de estrutura e processo, incluindo recursos educativos empregados com usuários e profissionais, disponibilidade de insumos, adequação da capacitação profissional e questões organizacionais de acessibilidade nos diversos tipos de UC. O conceito de "acesso potencial" incorpora fatores que interferem na capacidade de utilização dos serviços de saúde, ${ }^{23}$ e, no PTN, esses fatores incluem nível de informação das famílias, distância entre residências e UC, recursos financeiros desembolsados para realização do teste, entre outros.

O "acesso realizado" refere-se ao grau de mudança na saúde em relação ao volume de serviços consumidos. ${ }^{23}$ No caso do PTN-RJ, essa dimensão pode ser medida pela cobertura do programa, precocidade de início do tratamento e desfechos clínicos no longo prazo. Neste trabalho, observou-se que o acesso potencial foi limitado por fatores que restringiram o conhecimento das famílias sobre o teste e o acesso realizado pelas questões organiza- 
cionais e aspectos contextuais das UC.

Entre as dimensões de acesso propostas por Pechansky e Thomas, ${ }^{24}$ nesta avaliação, a dimensão "acolhimento" (como os serviços se organizam para receber os usuários e estes se adaptam às organizações) parece a mais afetada e prioritária para intervenções com o objetivo de melhorar o "acesso realizado". 23

O Institute of Medicine acrescentou a dimensão temporal ao acesso definindo-o como o uso, no momento oportuno, dos serviços. ${ }^{8}$ Nessa perspectiva, a avaliação mostrou que houve perda de oportunidades na realização dos testes e nas etapas subsequentes, com retardo no diagnóstico e tratamento.

Os PTN, com suas peculiaridades e complexidade, envolvem diversas etapas e atores e dependem dos sistemas de saúde em que estão inseridos. Potter et al. 25 analisaram os PTN e os sistemas de saúde dos Estados Unidos, Canadá, Inglaterra e Austrália e mostraram diferentes estratégias bem-sucedidas para envolvimento dos respectivos atores em cada país.

O Estado de Minas Gerais equacionou estas questões implementando a estratégia " 5 dias, 5 ações", divulgada durante o pré-natal. ${ }^{26}$ As gestantes comparecem aos serviços de saúde no $5^{\circ}$ dia após o parto para cuidados à puérpera e ao recém-nato que incluem TN. Assim, o PTN conseguiu alcançar excelente cobertura e coletas mais precoces.

A Secretaria Municipal de Saúde do RJ implementou a estratégia "Acolhimento mãe-bebê" 26 com encaminhamento das mães na alta da maternidade à unidade básica de saúde próxima à sua residência, onde mãe e recém-nato recebem cuidados básicos que incluem TN. Esta estratégia poderia ser reproduzida nos demais municípios do Estado do RJ.

Entre as limitações do estudo, estão: a) a necessidade de substituição de UC na amostra, levando ao predomínio dos postos de saúde do Estado, que é coerente com a sua maior capilaridade em todo o sistema de saúde do Estado do RJ; b) em algumas $\mathrm{UC}$, as observações foram comprometidas por restrições nos horários de coleta ou no acesso às geladeiras que armazenavam testes. Em termos de pontuação, isso não representou um problema, já que os escores destas UC foram recalculados de forma a não penalizá-las. Contudo, a limitação de horários para coleta é um importante fator de restrição ao acesso aos testes.

\section{Considerações finais}

Após mais de 20 anos do PTN-RJ, são evidentes e urgentes os ajustes para tornar o programa efetivo e apto a buscar novos horizontes de forma harmônica.
A inclusão de novas doenças torna imperativo que o processo ocorra rigorosamente dentro da janela de tempo ideal.

Os resultados desta avaliação mostram a necessidade de melhorar a capacidade gerencial e técnica das unidades de saúde, profissionais de saúde do prénatal, maternidades e UC, além de ampliar a informação aos usuários sobre o teste e implicações das doenças rastreadas.

Essa pesquisa sinaliza para alguns pontos que podem auxiliar nessa reflexão. Há que se pensar em um planejamento educativo com orçamento e metas definidos, capacitação e supervisão programadas conforme protocolo preconizado e as necessidades da rede. O material educativo deve estar adequado às diretrizes e direcionado a diversos públicos-alvo: associações de categorias profissionais envolvidas no pré-natal, coletas e busca ativa, gestores, usuários; inclusão nos currículos universitários e escolas técnicas. É importante considerar o caráter contínuo e permanente das medidas educativas, observando-se a natureza dinâmica e mutável desse sistema complexo que envolve atores e processos distintos.

Ainda que a coleta nas maternidades seja recomendável, no Estado do RJ há grande rotatividade de leitos, curta permanência dos recém-natos, altas hospitalares precoces e coletas tardias em unidades de saúde externas, além de estrutura inadequada para busca ativa. Para se evitar perdas de oportunidades e coletas tardias a assistência prénatal poderia incluir recomendações para triagem neonatal. A efetividade desta estratégia depende da aptidão dos profissionais que atendem as famílias após o nascimento para orientar sobre a realização do teste, e da capacitação e supervisão sistemática das equipes das UC para evitar coletas tardias e amostras de má qualidade que implicam em recoletas, retardam diagnósticos e afastam o PTN do seu objetivo.

Por fim, a avaliação com retroalimentação para os profissionais e gestores poderá contribuir para um melhor desempenho do Programa de Triagem Neonatal. Contudo, há que se investir também num acompanhamento mais rotineiro que produza informações que possam ser utilizadas para melhorar o programa e subsidiar decisões de forma rápida e oportuna.

\section{Agradecimentos}

A pesquisa foi realizada com apoio da FAPERJ (Processo APQ1 No E-26/111.443/2008) e do CNPq (Processo no 308651/2006-5). Agradecemos à 
Secretaria de Estado de Saúde do Rio de Janeiro, e às instituições e respectivas equipes que participaram na a pesquisa. Agradecimentos à Karla K. F.

\section{Referências}

1. Brasil. Ministério da Saúde. Portaria GM/no ${ }^{\circ}$. 822. Cria o Programa Nacional de Triagem Neonatal (PNTN). Diário Oficial da União. Brasília, DF, 7 jun 2001; 111(1).

2. Brasil. Ministério da Saúde. Secretaria de Assistência à Saúde. Manual de Normas Técnicas e Rotinas Operacionais do Programa Nacional de Triagem Neonatal. Brasília, DF; 2005. p. 128.

3. Therrell BL, Schwarz M, Southard C, Williams D, Hannon WH, Mann MY. Newborn screening system performance evaluation assessment scheme (PEAS). Semin Perinatol. 2010; 34: 105-20.

4. Health Resources and Services Administration, National Neonatal Screening \& Genetic Resources Center. Performance evaluation and assessment scheme for newborn screening systems. 2005. [acesso em 10 out 2006]. Disponível em: http://genes-r-us.uthscsa.edu.

5. Brasil. Ministério da Saúde. Portaria SAS no 491. Credencia o IEDE e APAE-RJ como Serviços de Referência em Triagem Neonatal. Diário Oficial da União. Brasília, DF, 23 out 2001

6. Brasil. Ministério da Saúde. Portaria SAS n ${ }^{\circ} 514$. Credencia a Secretaria Municipal de Saúde como Serviço de Referência em Triagem Neonatal. Diário Oficial da União. Brasília, DF, 5 nov 2001.

7. Botler J, Camacho LAB, Cruz MM. Análise do desempenho do Programa de Triagem Neonatal do Estado do Rio de Janeiro de 2005 a 2007. Cad Saúde Pública. 2011; 27: 2419-28.

8. Travassos C, Martins M. Uma revisão sobre os conceitos de acesso e utilização de serviços de saúde. Cad Saúde Pública. 2004; 20 (Supl. 2): S190-8.

9. Brasil. Ministério da Saúde. Secretaria de Assistência à Saúde - Grupo Técnico de Assessoria em Triagem Neonatal. Roteiro de cadastramento de Serviços de Referência em Triagem Neonatal nos Estados; 2001.

10. Am Ass Pediatr. Issues in newborn screening. Pediatrics. 1992; 89: 345-9.

11. Am Ass Pediatr. Update of newborn screening and therapy for congenital hypothyroidism. Pediatrics. 2006; 117: 22902303.

12. Ass Med Bras. Hipotireoidismo congênito. Projeto Diretrizes. 2005. [acesso em 2 mar 2010]. Disponível em: http://www.projetodiretrizes.org.br/4_volume/18Hipotiroid.pdf

13. Australasian Paediatric Endocrine Group. Position Statement. Guidelines for diagnosis, management and follow-up of children with congenital primary hypothyroidism. 1999. [acesso em 31 mai 2005]. Disponível em: http://www.racp.edu.au/apeg/position.htm
Costa pelas atividades de suporte de secretaria; e à Pâmela S. George e Constância J. C. Melo pelo trabalho de coleta de dados.

14. Health Council of the Netherlands. Neonatal Screening. 2005. [acesso em 15 jun 2006]. Disponível em: http://www.healthcouncil.nl

15. International Atomic Energy Agency. Screening for newborns for congenital hypothyroidism: guidance for developing programmes. Vienna; 2005. p. 122.

16. Newborn blood spot screening in the UK. Policies and standards. 2005. [acesso em 31 mai 2005]. Disponível em: http://www.newbornscreening-bloodspot.org.uk

17. Pass K, Lane PA, Fernhoff PM, Hinton CF, Panny SR, Parks JS, Pelias MZ, Rhead WJ, Ross SI, Wethers DL, Elsas-II LJ. US newborn screening system guidelines II: follow-up of children, diagnosis, management and evaluation statement of the Council of Regional Networks for Genetics Services (CORN). J Pediatr. 2000; 137: S1-6.

18. Therrell BL, Panny SR, Davidson A, Eckman J, Hannon WH, Henson MA, Hilard M, Kling S, Levy HL, Meaney FJ, McCabe ERB, Mordaunt V, Pass K, Shapira E, Tuerck J. US newborn screening system guidelines: Statement of the Council of Regional Networks for Genetic Services. Screening. 1992; 1: 135-47.

19. SPSS. Statistical Package for Social Sciences. Chicago, Illinois, United States; 2004

20. Hannon WH, Baily CM, Bartoshesky LE, Davin B, Hoffman GL, King PP, Neier SS, Peter JA, Therrell, BL. Blood collection on filter paper for newborn screening programs; approved standard - fourth edition. NCCLS document LA4-A4. 2003; 23(21): 27.

21. Botler J, Camacho LAB, Cruz MM. Phenylketonuria, congenital hypothyroidism and haemoglobinopathies: public health issues for a Brazilian newborn screening program. Cad Saúde Pública. 2012; 28: 1623-31.

22. Rovet J, Daneman D. Congenital hypothyroidism: a review of current diagnostic and treatment practices in relation to neuropsychologic outcome. Paediatr Drugs. 2003; 53: 1419

23. Andersen RM. Revisiting the behavioral model and access to medical care: does it matter? J Health Soc Behav. 1995; 36: $1-10$.

24. Pechansky R, Thomas JW. The concept of access - definition and relationship to consumer satisfaction. Med Care. 1981; 19: 127-40

25. Potter BK, Avard D, Wilson BJ. Newborn blood spot screening in four countries: stakeholder involvement. J Publ Health Pol. 2008; 29: 121-42.

26. Minas-Gerais. Secretaria Estadual de Saúde. Atenção à Saúde da Criança; 2005. [acesso em 7 jan 2012]. Disponível em http://www.saude.mg.gov.br/publicacoes

Recebido em 16 de maio de 2012

Versão final apresentada em 14 de agosto de 2012

Aprovado em 25 de setembro de 2012 